\title{
Voltage Noise Induced DRAM Soft Error Reduction Technique for 3D-CPUs
}

\author{
Tiantao Lu, Caleb Serafy, Zhiyuan Yang and Ankur Srivastava \\ University of Maryland, College Park, MD, USA \\ \{ttlu, cserafy1, zyyang, ankurs\}@umd.edu
}

\begin{abstract}
Three-dimensional integration enables stacking DRAM on top of CPU, providing high bandwidth and short latency. However, non-uniform voltage fluctuation and local thermal hotspot in CPU layers are coupled into DRAM layers, causing a nonuniform bit-cell leakage (thereby bit flip) distribution. We propose a performance-power-resilience simulation framework to capture DRAM soft error in 3D multi-core CPU systems. A dynamic resilience management (DRM) scheme is investigated, which adaptively tunes CPU's operating points to adjust DRAM's voltage noise and thermal condition during runtime. The DRM uses dynamic frequency scaling to achieve a resilience borrow-in strategy, which effectively enhances DRAM's resilience without sacrificing performance.
\end{abstract}

\section{INTRODUCTION}

Three-dimensional integration of stacked memory and CPUs has received many attentions recently for its potential to overcome the "Memory Wall" problem - the memory access time (in CPU cycles) has increased to an extent that the memory access latency has become the bottleneck. Vertical stacking enables heterogeneous integration of multiple DRAM chips on top of a microprocessor, and the high-speed and wider memory bus interfaces (i.e. through-silicon-vias) between the two significantly reduce memory latency.

However, several recent publications have shown that transient fault is one of the common forms of DRAM failures in modern computing and storage systems. One important cause of DRAM transient faults is the power-delivery-network (PDN) noise on DRAM wordline (WL). A noisy WL makes DRAM transistor's gate unable to shut off, causing significant subthreshold leakage from bit cell's capacitor. As volatile memory, a DRAM bit cell cannot retain its data permanently as the bit capacitor gradually loses its charge. If significant leakage occurs such that DRAM sense amplifier can no longer read the correct data as written last time, a DRAM transient fault happens.

The architectural solutions for mitigating stacked DRAM's transient fault seem non-trivial. An intuitive idea is to enable shorter DRAM refresh period. However, refreshing operation blocks DRAM access, therefore degrades the performance and energy efficiency $[13,3]$. Another approach is to throttle CPU performance to cool the chip or/and reduce PDN noise, which again hurts the performance. In our opinion, a promising opportunity lies at the moment when both low and high power tasks enter the system, and designers can exploit the reliability margin of these tasks and allocate different operating points to maximize the performance while achieving certain long-term resilience. A "borrow-in" strategy can be applied, and high-power tasks might borrow resilience margins to boost performance, and the resilience "loan" will be paid off during a low-power task period.

Permission to make digital or hard copies of all or part of this work for personal or classroom use is granted without fee provided that copies are not made or distributed for profit or commercial advantage and that copies bear this notice and the full citation on the first page. Copyrights for components of this work owned by others than ACM must be honored. Abstracting with credit is permitted. To copy otherwise, or republish, to post on servers or to redistribute to lists, requires prior specific permission and/or a fee. Request permissions from permissions@ acm.org.

ISLPED '16, August 08-10, 2016, San Francisco Airport, CA, USA

(c) 2016 ACM. ISBN 978-1-4503-4185-1/16/08 . .\$15.00

DOI: http://dx.doi.org/10.1145/2934583.2934589
To the best of our knowledge, although there have been many field studies about the transient DRAM fault recently [17, $23,28]$, simulation frameworks that investigate its underlying causes, its implications in 3D CPUs, and corresponding mitigation techniques have been lacking. Several publications use fault-injection technique based on constant DRAM failure rate to investigate DRAM resilience $[11,21]$, however, the fact that different CPU workload causes a temporal and spacial DRAM fault rate variation has been ignored. In 3D CPU architectures, CPU activity causes fluctuation in PDN voltage and temperature, both of which gets coupled into DRAM layers. The integration of multi-dimensional inter-dependent simulation analysis (performance, power, thermal and voltage) is a challenging task. A more fundamental dilemma for simulation setup is the gap between CPU cycle-level performance / power simulation and long-term DRAM resilience simulation. Although CPU performance / power simulation is crucial in determining the thermal and PDN transients, it's not practical to perform cycle accurate simulation across the entire DRAM lifetime (often in years). This gap needs to be filled for accurate DRAM resilience analysis within practical run-time.

In this paper, we focus on the WL voltage noise induced stacked DRAM transient fault problem. Although there are other transient fault causes, such as particle-induced upset, they are heavily investigated in the literature, and their trends seem irrelevant with $3 \mathrm{D}$ integration. We propose a performance - power - voltage - resilience simulation framework, which effectively captures the interaction between multi-core CPU layer's activity, and voltage noise induced DRAM transient fault. We observe significant correlation between CPU core's activity and DRAM voltage and thermal profiles, both in time and space. As the DRAM leakage strongly depends on voltage noise and temperature, we further observe significant temporal and spacial variation in bit-cell's leakage rate. These two observations imply that the DRAM failure probability strongly depends on 3D CPU's operating points. In our simulation experiments, we investigate an off-line task assignment problem, and propose a two-stage DRAM resilience management approach, which enables frequency allocation for CPU cores and refresh rate adjustment for DRAMs, in order to optimize 3D-CPU's performance while accommodating DRAM resilience requirement.

Specifically our contributions are summarized as follows:

1. Contrary to common assumptions that DRAM transient error is random in time and space, we demonstrate that stacked DRAM's transient error is strongly correlated with 3D CPU activities, through voltage and thermal coupling.

2. Using SPICE simulation, we show that DRAM bit cell leakage is an accumulative effect, indicating it's more related to IR drop rather than transient droop.

3. We propose an effective off-line operating point tuning approach to solve a task assignment problem, which allocates frequency and refresh rate for CPU cores and DRAM controllers, such that the system's performance is maximized, while ensuring DRAM resilience. Compared to a simplistic frequency throttling method, significant performance enhancement is achieved.

\section{RELATED WORK}


In this section, we provide a brief overview of recent work on DRAM transient error field experiment and simulation, and we also cover prevailing management methods.

\subsection{Background: DRAM Transient Fault}

Transient fault in DRAM is a very common form of hardware failure in modern computing clusters and data centers. DRAM's transient fault can be caused by voltage noise and external particles (i.e. alpha particles and neutrons). Voltage noise on the WL causes transistor sub-threshold current and loss of charges on the bit capacitor. The sources of external particles that include alpha particles emitted by packaging materials, and neutrons from cosmic radiation [2].

Recently, several major data servers have shown field experimental studies for detecting and measuring DRAM transient fault. Google [23], AMD[28], and Facebook [17] have reported significant transient error rate during years-long studies.

Statistical modeling approaches such as Monte Carlo (MC) methods are adopted to analyze the probability of DRAM failure under different resilience schemes $[11,21]$. The lifetime of DRAM into equal-sized time intervals and transient faults are injected into memory array based on their probability of occurrence. Error detection and correction are invoked periodically to determine whether the injected faults are correctable or not.

In 3D-CPU architectures, power is delivered from external power sources to CPU and DRAM layers, therefore, CPU activity has a strong impact on DRAM layer's voltage fluctuation. An active CPU core draws more current from PDN, causing higher IR drop (and possibly more Ldi/dt voltage droop) in the DRAM layer. We are especially interested in the voltage noise on DRAM WL, as it causes sub-threshold leakage over time. In summary, this paper focuses on the WL voltage noise induced DRAM transient faults.

\subsection{Existing Mitigation Techniques}

A variety of architectural techniques exist to mitigate the DRAM transient fault problem. The most effective and prevailing method is hardware-based parity or ECC [5]. Parity is typically an XOR of all data bits and is able to detect any single-bit error. As a more powerful scheme, ECCs such as ChipKill [6] and $\mathrm{BCH}$ [7] enable detection and correction of one or multiple bit errors. Hardware-based ECC usually has certain hardware overhead, for example, $\mathrm{BCH}$ has an overhead of 8 bits per 64 bits of data.

Reducing the refreshing time interval is an intuitive method to mitigate DRAM's transient fault. However, memory refresh wastes energy and degrades performance by interfering with memory access $[13,3]$. The energy and performance overheads of DRAM refresh increase as DRAM device capacity expands.

Since DRAM transient faults can accumulate over time, another option is to perform memory scrubbing that scans throughout the memory and then write back the corrected bit information before single-bit fault mounts to multi-bit uncorrectable fault [19]. However, such a heavy task also incurs significant performance and energy overhead.

Software-based technique includes completely mirroring CPUs or threads. Identical copies of the same program are run simultaneously and the operating system can determine the correct data based on majority voting $[27,1,18]$.

Another possibility is to change the operating point for dynamic reliability management. Mercati etc. proposed a sensor based CPU reliability control scheme, where voltage and frequency operating points are dynamically allocated to improve the performance while meeting the long-time CPU reliability constraint [16]. Shevgoor etc. proposed an IR-drop-aware memory controller and a page migration method to cope with IR-drop constraint[25]. While these approaches are effective in handling CPU core reliability degradation, their implications on DRAM resilience are unclear.

\section{SIMULATION FRAMEWORK}

In order to analyze the correlation between 3D CPU activity and stacked DRAM resilience, an integrated analysis of how CPU's performance and power transients influences DRAM layer's thermal and voltage behavior is of vital importance. One also needs to quantify how WL noise induces bit flip. Our performance / power / voltage / resilience simulation and optimization flow is summarized in Fig.1.

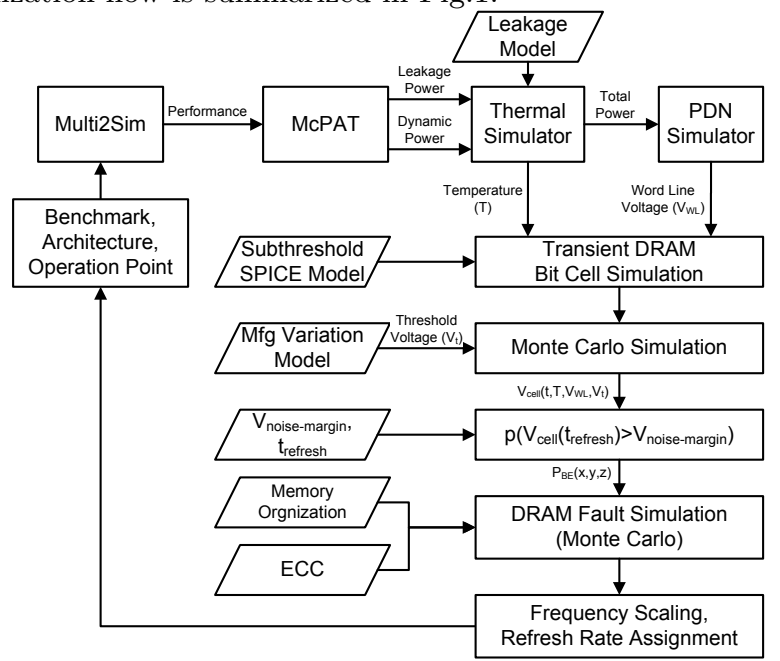

Figure 1: Overview of Performance / Power / Voltage / Resilience Simulation and Optimization flow for 3D-CPU.

Multi2Sim is used to produce performance data (instructions executed per nanosecond) and number of memory access[29]. Dynamic and leakage powers of 3D-CPU are simulated with McPAT [12]. The power data are fed into in-house thermal and PDN simulator to generate transient thermal and WL voltage noise distribution. This is followed by a DRAM bit cell SPICE simulation, which captures the sub-threshold leakage current within one DRAM refresh period. Once the noise margin of DRAM bit information is known, we use MC simulation to obtain the probability of a bit cell error $\left(P_{B E}\right)$, for a given random manufacturing variation. The $P_{B E}$ value is a function of three dimensional space, which is the input of a DRAM resilience simulator. The DRAM resilience simulator maps the $P_{B E}$ value into memory space, and a high $P_{B E}$ value at certain $(x, y, z)$ location will have a higher probability of transient error in its corresponding memory address space. ECC scheme such as SECDED is evoked periodically. The DRAM fault simulator returns the probability of uncorrectable errors after thousands of MC iterations. After all the benchmarks and all the operating points have been profiled, a feedback loop is created to adjust the operating points of $3 \mathrm{D}$-CPUs. Specifically we consider CPU core frequency scaling and DRAM refresh rate adjusting as two tuning knobs for optimizing CPU performance and DRAM resilience.

\subsection{Performance/Power/Thermal/Voltage Simu- lator}

\subsubsection{Performance Simulation}

Performance of a software workload on a target 3D CPU is estimated using Multi2Sim [29], a cycle-accurate multi-core CPU simulator. Architectural parameters of the target system including memory and cache architectures and latencies, operating frequency, network-on-chip (NOC) topology, pipeline width, function unit count etc. are given to the simulator and the simulator reports the total run time, number and type of executed instructions, and access counts of the various CPU components. The performance metric considered in this work is the number of instructions executed per nanosecond (IPnS).

\subsubsection{Power/Area Estimation}

Power estimates are calculated using McPAT [12]. The tool considers the architectural parameters of the target system and the component access counts generated by the cycle-accurate performance simulator (Sec. 3.1.1). The architectural parameters are used to determine the energy-per-access and total area of each CPU components. The performance counters determine the number of accesses to each component, thus yielding the total energy consumption and average power consumed during the simulation period. The estimation tool outputs a hierarchi- 
cal list of dynamic and leakage power estimates for each CPU component. The power distribution is used to analyze the chip temperature (Sec. 3.1.3) and IR drop (Sec. 3.1.4).

\subsubsection{Thermal Analysis}

A 3D grid thermal resistance model of the target 3D CPU is generated based on dimensions and material properties. The power located in each grid is modeled by a current source. This technique is similar to other proposed thermal simulation techniques such as HotSpot [9]. The generated circuit model is solved to yield a voltage distribution which is representative of the thermal distribution of the target 3D stack. Thermal estimates are used to re-estimate leakage power using the baseline estimates from McPAT (which assumes a uniform user-selected temperature) and a thermal-leakage scaling model extracted from the McPAT source code [24]. This thermal-leakage feedback repeats until the convergence. Final thermal estimates are used to model the DRAM bit cell leakage rates and the associated probability of bit errors (Sec. 3.2).

\subsubsection{PDN Modeling}

Our PDN model is an RLC circuit which includes on-chip and off-chip components. Each on-chip grid is connected to its neighbor through a resistor and a inductor in series, and is also attached to a current load. On-chip decoupling capacitance is distributed uniformly on-chip. Power/ground C4 bumps are modeled as series of resistors and inductors between on-chip and off-chip networks. Power/ground TSVs establish vertical power delivery. We only model the power rail because the ground rail has symmetric property.

\subsection{DRAM Bit Flip Probability Modeling}

Noise induced DRAM bit cell's failure probability is estimated by MC simulation and a transistor level SPICE simulation. During one DRAM refresh period, WL noise induces subthreshold leakage current, which causes charge transfer from the bit-line capacitor to the DRAM capacitor. Once the change of charges exceeds the noise margin, a bit fault occurs.

We assume DRAM manufacturing process causes random variation in bit-cell transistor's gate oxide thickness. A transistor with thin gate oxide leaks faster, and the probability of bit flip is calculated by the total number of flipped bit divided by the number of MC simulations.

The transistor leakage model, Eqn. (1a), is from [22]. The temperature-dependent leakage scaling model, Eqn.(1b) is extrapolated from the McPAT [12] source code, as in [24].

$$
\begin{gathered}
I(t)=I_{0}(T(t)) e^{\left(V_{g s}(t)-V_{t h}\right) q / n k T(t)}\left(1-e^{\left(-V_{d s}(t) q / k T(t)\right)}\right) \\
I_{0}(T(t))=I\left(T_{0}\right) \times\left(5.121 \frac{T(t)^{2}}{T_{0}}-6.013 \frac{T(t)}{T_{0}}+1.892\right) \\
\frac{d V_{\text {cell }}(t)}{d t}=\frac{I(t)}{C_{\text {cell }}}
\end{gathered}
$$

where $I$ is DRAM transistor leakage, $t$ is time, $T$ is temperature, $V_{g s}$ is gate-to-source voltage, $V_{t h}$ is threshold voltage, $q$ is electron charge, $k$ is Boltzmann constant, $V_{d s}$ is drain-to-source voltage, $T_{0}$ is nominal temperature, $V_{\text {cell }}$ is bit-cell's voltage, and $C_{c e l l}$ is cell capacitance.

Fig.2(a) shows the transient of voltage and temperature changes in DRAM layers during the first $32 \mathrm{~ms}$ refresh period when executing benchmark "bodytrack". Oscillation in CPU activity is coupled into the DRAM layer, causing thermal and voltage fluctuations. The transient of bit-cell voltage (with WL noise) is shown with the solid black line in Fig.2(b). The supply voltage is 1 Volt. The transient voltage and temperature data in Fig.2(b) are sampled at $280 \mu s$, and the adaptive time step used by the DEQ solver to calculate bit cell voltage is roughly 10x small than the data sampling rate. For comparison, an ideal WL voltage (perfectly grounded) is applied for the same bit-cell, and the voltage on its capacitor is plotted with the black dotted line. As can be seen in the figure, the original bit information " 0 " gets corrupted much fast due to WL noise.

Another transient is performed with the average voltage noise and temperature as inputs, and the bit-cell's voltage trace is solved similarly. We refer this as "V-T steady state" simulation. The resulting bit cell voltage is plotted with the dashed black curve. The "V-T steady state" simulation converges with the real transient simulation at the end of the refresh cycle. Note that in Eqn.(1), the leakage current is an exponential function of $V_{g s}$, where $V_{g s}=V_{W L}-V_{C e l l}$. However, the reason that "V-T steady state" simulation achieves high accuracy is that within the temperature and voltage noise range $\left(35^{\circ} \mathrm{C}-45^{\circ} \mathrm{C}\right.$ and around $100 \mathrm{mV}$ WL noise), the bit-cell voltage as function of temperature and WL noise is operating at a linear region. This effect has important implications on operating point tuning techniques such as dynamic frequency and voltage scaling (DVFS). When the time constant of DVFS is in the same order of magnitude with DRAM refresh period (V-F selection in roughly every $50 \mathrm{~ms}$ in [15]), taking the average of voltage noise and temperature and then simulate the transient of bit cell voltage will be fast and accurate.

We also perform two sensitivity analyses, one with ambient temperature and transient voltage noise, and another with transient temperature and ideal WL voltage (i.e. grounded). The results are plotted with blue dashed line and red solid line, respectively. The data show that when executing "bodytrack", WL noise seems to be a more dominating factor than temperature for sub-threshold leakage.

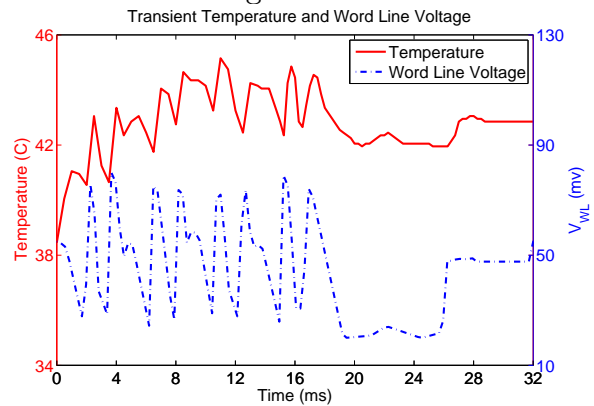

(a)

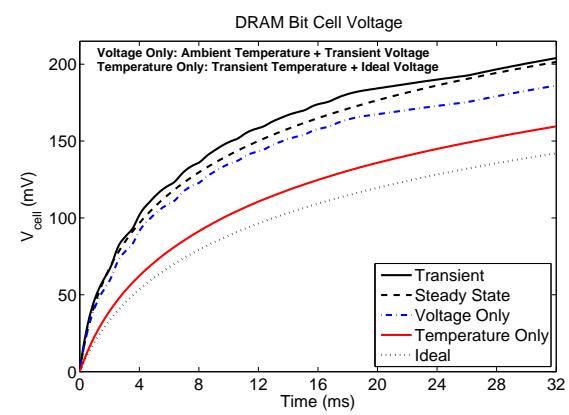

(b)

Figure 2: (a) Transient WL noise and temperature change in DRAM layer during a $32 \mathrm{~ms}$ refresh cycle of "bodytrack" execution. (b) DRAM bit transistor's sub-threshold leakage charges the bit capacitance over time. The original bit information " 0 " gets corrupted when capacitor's voltage exceeds the noise margin.

\subsection{DRAM Resilience Simulator}

The DRAM resilience simulator uses MC method to obtain the probability of DRAM failure under a given ECC scheme. Based on DRAM layer's thermal and voltage noise distribution generated by thermal and PDN simulations for a particular CPU activity transient, we obtain the physical distribution of $P_{B E}$. A DRAM block with a thermal hotspot and a noisy WL has higher probability of bit flip. A random number of bit flips per grid is generated according to binomial error distribution. The spacial distribution of bit flip numbers is then mapped into memory address space, for a given memory organization and physical layout. We use the data structure of Faultsim [21] to represent transient faults in memory address space. Finally ECC is evoked and the simulator returns whether the voltage noise induced transient fault causes an uncorrectable DRAM 
fault. Thousands of MC simulations are performed with random manufacturing variation until a reliable probability of uncorrectable DRAM failure $(P O F)$ is obtained.

\section{DRAM RESILIENCE MANAGEMENT}

Using the simulation framework discussed in Sec. 3.1 we are able to obtain the spatial distribution of $P_{B E}$ and the corresponding $P O F$ when executing different phases of a set of software workloads. With this profiling data we are able to estimate the optimal core frequency and DRAM refresh rate for each phase of each application using the scheme shown in Fig. 3.

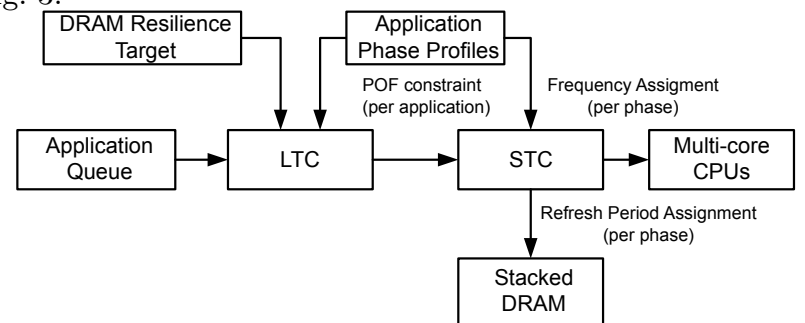

Figure 3: Control scheme for frequency and refresh rate assignment across applications (long term control) and application phases (short term control).

In Fig.3, the optimization goal is to maximize the system performance while meeting a specified DRAM resilience target (i.e. the long-term probability of an uncorrectable memory error). The long-term period is divided into several short-term periods. The long term controller (LTC) takes a set of applications awaiting execution from the application queue, and determines at which operating point (i.e. frequency/refresh rate setting) each application should be run to minimize runtime while meeting the long term DRAM resilience target $R_{t}$ averaged across all considered applications. The short term controller (STC) optimizes the runtime of each application by assigning an operating point to each phase of the application. The $P O F$ estimate output by LTC serves as a constraint on the POF of the application during STC optimization.

One limitation of our control scheme is it relies on the availability of profile data, which assumes that the workloads can be predicted off-line. While this assumption may not be applicable to all systems, it applies well to embedded systems [10] and high performance computing (HPC) systems [26]. These are two paradigms where the stacked DRAM processor shows great potential due to its small form factor and low power (embedded systems) and/or massive memory bandwidth (HPC).

\subsection{Long Term Controller}

During one long term period, we assume DRAM bit errors do not accumulate from one application to the next. Any errors accumulated in one application are effectively corrected when the application completes and its memory is deallocated. Thus the $P O F$ of each application is independent and the DRAM resilience $(R)$ is calculated using Equation (2).

$$
R=\prod_{i}\left(1-P O F_{i}\right)
$$

The DRAM resilience constraint can be linearized using Equation (3), allowing the assignment problem to be formulated as an integer linear program (ILP) in Equation (4). $\delta_{i, j, k}$ is a binary variable such that it is set when application $i$ is assigned to run at frequency $j$ and DRAM refresh rate $k$. Likewise $t_{i, j, k}$ and $P O F_{i, j, k}$ are the runtime and $P O F$ of application $i$ running at frequency $j$ and DRAM refresh rate $k$.

$$
\prod_{i}\left(1-P O F_{i}\right) \geq R_{t} \rightarrow \sum_{i} \ln \left(1-P O F_{i}\right) \geq \ln \left(R_{t}\right)
$$

Decision variable : $\delta_{i, j, k}$

$$
\begin{array}{ll}
\max & \sum_{i, j, k} \delta_{i, j, k} \\
\text { s.t. } & \text { 1. } \quad \sum_{i, j, k} t_{i, j, k} \cdot \delta_{i, j, k} \leq t_{L T C} \\
& \text { 2. } \quad \sum_{i, j, k}-\ln \left(1-P O F_{i, j, k}\right) \cdot \delta_{i, j, k} \leq-\ln \left(R_{t}\right) \\
& \text { 3. } \quad \sum_{j, k} \delta_{i, j, k} \leq 1 \forall_{i} \\
& \text { 4. } \quad \sum_{j, k} \delta_{i, j, k}-\sum_{j, k} \delta_{i-1, j, k} \leq 0 \forall_{i}
\end{array}
$$

The ILP problem objective maximizes the number of assigned applications and Constraint 1 ensures that the total runtime of all assigned applications is less than the LTC control window $t_{L T C}$. By maximizing the number of applications assigned in a fixed period of time we maximize performance since applications must be executed in order. Constraints 3 and 4 respectively ensure that each application is assigned no more than one operating point and applications are executed in order. Constraint 2 uses the linearized resilience constraint from Equation (3) to generate operating point assignments that meet the resilience constraint across the LTC control window.

The assignment of operating points to each application yields an expected $P O F$ and runtime value for each application such that total runtime is minimized and the DRAM resilience target is met. However the assignment of a single operating point to each application is overly constrained. We improve our assignment solution by allowing different operating points to be assigned to each phase of a single application using the short term controller such that the application's $P O F$ is less than the $P O F$ estimated by the LTC but the total runtime is reduced.

\subsection{Short Term Controller}

The short term controller (STC) allocates operating points to each phase of one application. The $P O F$ estimate output by LTC is a $P O F$ constraint during STC optimization. Within a single application DRAM transient fault from phase to phase do compound, which means the $P O F$ of an application cannot be described as a linear combination of the $P O F$ of each phase. The problem could be solved by expanding the size of the ILP problem to include all possible sequences of operating points, but this would be computationally infeasible for nontrivial problem sizes. Moreover the profiling effort to generate $P O F$ values for all combinations of operating points would be unnecessarily detailed. So we instead solve the STC optimization problem using a simulated annealing (SA) heuristic. Since the initial solution given by the LTC (i.e. run all phases of one application at the given operating point) is already a good solution, low temperature SA does well to improve the solution.

The annealing function used in our SA formulation moves the operating point of one phase to a neighboring operating point (i.e. increase or decrease the frequency or refresh rate by one level). The SA objective function evaluates the runtime and $P O F$ of each considered sequence of operating points and evaluates Equation (5). $r_{i}$ is the runtime of phase $i$ running at the assigned operating point. $P O F$ is the $P O F$ of the entire application running at the considered sequence of operating points. Evaluation of $P O F$ is explained below. $P O F_{L T C}$ and $R T_{L T C}$ are the $P O F$ and runtime estimate generated by the LTC for this application, assuming all phases were run at a single operating point.

$$
O B J=c_{1} \cdot \frac{\sum_{i} r(i)}{R T_{L T C}}+c_{2} \cdot \max \left(1, \frac{P O F}{\sum_{i} r(i)} / \frac{P O F_{L T C}}{L T_{L T C}}\right)
$$

The first objective term tries to reduce the total runtime of an application. However if the runtime of an application is reduced the probability of failure must reduce by the same amount to maintain to long term $P O F$ constraint. The second term serves to constrain the optimization by assigning a large penalty if the considered ratio between $P O F$ and runtime in- 
creases above the one assigned by the LTC. Since the second term is a constraint, $c_{2}$ is much greater than $c_{1}$.

Note that the runtime of each phase at each operating point is already available in our profile data, and we can simply sum up all phases to evaluate the runtime of the entire application.

\subsection{Fast Conversion from $P_{B E}$ to $P O F$}

In Eqn.5, the evaluation of the POF (of an entire application) requires simulating the sequence of associated $P_{B E}$ distributions using our DRAM resilience simulator (Sec. 3.3), which takes significant runtime. In order to speed up the optimization we propose a fast conversion method to convert a sequence of $P_{B E}$ distributions into a $P O F$ estimation, based on the observation in Fig. 4.

Fig. 4 shows a plot of the $P O F$ value estimated from our DRAM resilience simulator versus the average $P_{B E}$ over $3 \mathrm{D}$ space. As the average $P_{B E}$ increases, more bit flips occur therefore it becomes harder for ECC detection. Each red data point represents running one benchmark at a specific combination of core frequency and DRAM refresh rate. We observe that the data fits an error function very well, and conclude that such the fitted function can be used to convert an arbitrary average $P_{B E}$ over space into a scalar $P O F$ value. The fitted error function is shown in Fig. 4.

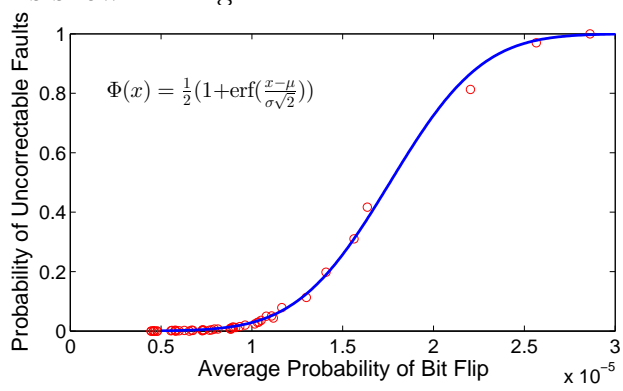

Figure 4: Probability of uncorrectable fault $(P O F)$ versus probability of bit flip $\left(P_{B E}\right)$ averaged over three dimensional space.

The data in Fig. 4 is derived using the DRAM resilience simulator with a specific $P_{B E}$ distribution. In our SA objective function we must convert a sequence of $P_{B E}$ distributions to the application's $P O F$, since each application consists of multiple phases with varying operating points. We deduce that a sequence of $P_{B E}$ distributions can be converted to an equivalent single distribution by taking the maximum value across all members of the sequence for each point in the spatial distribution. Because the underlying source of randomness (i.e. manufacturing variations) is not changing over time, it is in fact the phase with the highest $P_{B E}$ that determines if bit flip occurs at that point. If the bit error does not occur when the probability is the highest (i.e. when the leakage current is the highest) it is not possible that the bit error would have occurred when the probability was less (i.e. when the leakage current was lower). Thus to evaluate an application's $P O F$, we first generate an equivalent $P_{B E}$ distribution by taking the maximum $P_{B E}$ across all members of the sequence at each point in space, and then evaluate $P O F$ based on the mapping in Fig.4.

\section{SIMULATION RESULTS}

\subsection{Experimental Setup}

We study a 3D CPU architecture with four layers of DRAM (4GB total capacity) and one layer of CPU. Each DRAM layer represents one rank. One DRAM rank is partitioned into four banks, and within each bank, there are 131072 rows and 16384 columns. Each DRAM rank is connected to the CPU layer with a 512 bit bus implemented with TSVs. The memory organization we consider is similar to previous stacked DRAM publications $[14,15]$. Distributed refresh mode supported by JEDEC is used. The CPU layer containing 16 cores is stacked above the DRAM layers and adjacent to the heat sink to prevent thermal violations. We mesh the $3 \mathrm{D}$ CPU into multiple grids, with the size of $200 \mu \mathrm{m} \times 200 \mu \mathrm{m}$ for each grid. As the power estimates provided by McPAT are at the function unit granularity which is on the order of $0.1 \mathrm{~mm}^{2}$, the $200 \mu \mathrm{m} \times 200 \mu \mathrm{m}$ grid size is sufficient to capture the thermal and voltage variance in both the multi-core processor and DRAM layers. BCH [7] is used as ECC.

For PDN modeling, the on-chip decoupling capacitance density is $33 \mathrm{nF} / \mathrm{cm}^{2}$ [8]. Other parameters of PDN is calculated based on its physical dimension and material property, similarly to the techniques proposed in [20].

For DRAM bit flip probability $P_{B E}$ modeling, we assume a normal distribution of gate oxide thickness. The DRAM capacitance is $30 \mathrm{fF}$. The DRAM cell's noise margin depends on the strength of the DRAM sense amplifier, and here we assume that a bit error occurs if the bit cell voltage degrades more than $10 \%$ VDD from ideal. For a given DRAM refresh period, a hundred thousand MC simulations are run for each WL voltage and temperature point to obtain accurate $P_{B E}$ distribution. The run time of the total MC simulations is about 12 hours for a PC with an Intel Core i5 3.1GHz CPU and $12 \mathrm{~GB}$ RAM. We only need to run the MC simulations once to obtain $P_{B E}$ as a function of PDN noise and temperature.

We use part of SPLASH-2 and PARSEC benchmarks in our simulation $[30,4]$. These benchmarks are commonly used to profile highly parallel computing systems, and we assume them to be representative of the different computing operations to be encountered in real applications. We profile our proposed control scheme across a sequence of applications, where each application is composed of different computing phases which are represented by the benchmarks. Each application is randomly assigned a nominal runtime (i.e. runtime if it were assigned the nominal operating point) and a random distribution of how much runtime is consumed by each type of phase. It is likely that a single application only contains a subset of all possible phases, and even applications containing the same subset can have drastically different distributions of runtime across phases. The specific benchmarks we use are: bodytrack, dedup, $\mathrm{fft}$, fluidanimate, radix and swaptions.

Performance, power, voltage and temperature for each benchmark at each operating point are generated using the simulation framework described in Section 3.1. DRAM refresh rate affects memory latency due to less availability of the DRAM. Memory latency is scaled from the nominal value proportional to the increase in DRAM overhead (refresh time divided by refresh period). Although the DRAM refresh rate determines the DRAM memory latency, it affects performance differently for different benchmarks as a function of their memory usage: memory bound workloads will gain a large performance improvement if memory refresh period is increased whereas CPU bound workloads will see little improvement.

We set the long term control window $\left(t_{L T C}\right)$ to 30 minutes and assign application runtime uniformly between 1.33 to 8 seconds. The $P O F$ constraint within a long term control window is $0.3 \%$. The controller assigns each phase of each application a frequency value from the set $\{3.0,2.5,2.0,1.5\} \mathrm{GHz}$ and a refresh period from the set $\{8,12,16,32,64\} \mathrm{ms}$.

\subsection{Results}

We analyze the distribution of operating points assigned to our controller across a set of 520 applications. The nominal operating point is $3.0 \mathrm{GHz}$ clock frequency and $8 \mathrm{~ms}$ refresh period. The results of this analysis are shown in Table 1. Although 20 combinations of frequency and refresh are possible, 13 of them are never assigned. The 32/64 ms refresh rates are never chosen by the scheduler. This is because the DRAM IR drop can be as high as 0.1 VDD (shown in Fig.2(a)), which leads to significant increases to DRAM transistor leakage and that is why $32 / 64 \mathrm{~ms}$ are used for $2 \mathrm{D}$ ICs but only shorter refresh periods are feasible for 3D ICs.

Fig. 5 plots the 12 operating points $(32 / 64 \mathrm{~ms}$ refresh rates are excluded in this figure) and their corresponding runtime and $P O F$ for a representative application from the optimized set. It can be seen that four operating points for this application are not on the Pareto optimal front, and thus will never be chosen by the controller. This explains the data in the upper right corner of Table 1 . In this distribution the nominal 


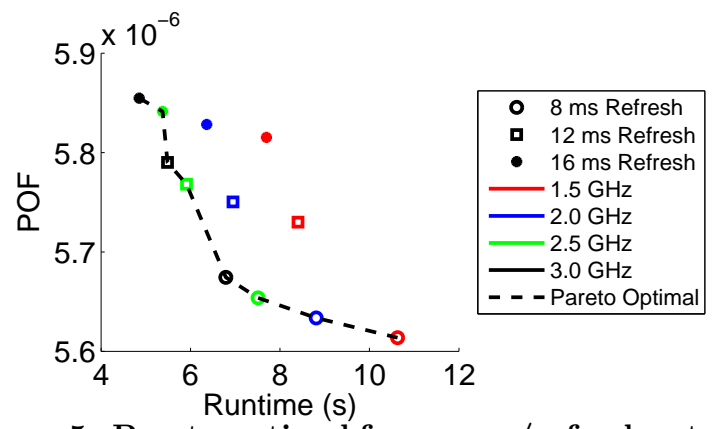

Figure 5: Pareto optimal frequency/refresh rate points for a representative application.

operating point is chosen a majority of the time, but significant throttling of both frequency and refresh rate are used to optimally trade off performance and DRAM resiliency. For example, an application with high power (which causes PDN noise and temperature increase) may require frequency to be reduced, whereas a lower power application puts less stress on the DRAM resiliency constraint and can be configured to run with a longer refresh period and get a performance boost

Table 1: Distribution of operating points assigned by our controller

\begin{tabular}{|c|c|c|c|c|c|c|}
\hline & \multicolumn{5}{|c|}{ Refresh Rate } \\
\hline & & $8 \mathrm{~ms}$ & $12 \mathrm{~ms}$ & $16 \mathrm{~ms}$ & $32 \mathrm{~ms}$ & $64 \mathrm{~ms}$ \\
\hline \multirow{4}{*}{ Freq. } & $1.5 \mathrm{GHz}$ & $1.8 \%$ & $0.0 \%$ & $0.0 \%$ & $0.0 \%$ & $0.0 \%$ \\
\hline & $2.0 \mathrm{GHz}$ & $1.8 \%$ & $0.0 \%$ & $0.0 \%$ & $0.0 \%$ & $0.0 \%$ \\
\hline & $2.5 \mathrm{GHz}$ & $7.2 \%$ & $0.0 \%$ & $0.5 \%$ & $0.0 \%$ & $0.0 \%$ \\
\hline & $3.0 \mathrm{GHz}$ & $59.3 \%$ & $27.5 \%$ & $2.0 \%$ & $0.0 \%$ & $0.0 \%$ \\
\hline
\end{tabular}

We compare the performance subject to $P O F$ constraint of our control scheme to the baseline case of running at nominal frequency and refresh rate. Our technique is able to improve system throughput (jobs completed per second) by $27 \%$. Our controller improves performance in two ways: 1) it allows applications to trade off resiliency and performance with one another and 2) it allows slack in the POF constraint to be converted to performance improvements.

Table 2: Throughput improvement using our frequency/refresh rate controller scheme

\begin{tabular}{|l|cc|}
\hline & Optimal & Nominal \\
\hline POF & $0.30 \%$ & $0.23 \%$ \\
Throughput (jobs/second) & 0.29 & 0.23 \\
\hline Normalized Throughput & 1.27 & 1.00 \\
\hline
\end{tabular}

\section{CONCLUSION}

This paper presents a detailed analysis and optimization scheme for 3D CPUs on voltage noise induced DRAM transient fault. Significant correlations between CPU activities and DRAM layer thermal and voltage noise behaviors have been observed. We show that under certain DRAM resilience target, the currently off-the-shelf DRAM refresh period (32 or $64 \mathrm{~ms}$ ) is not sufficient, however arbitrarily applying faster DRAM refresh rate inevitably hurts the performance. We propose a dynamic DRAM resilience management technique, which tunes CPU frequency and DRAM refresh rate in order to maximize performance while meeting long-term resilience target. Simulation results show that our management scheme achieves $27 \%$ increase in performance when comparing to running at the nominal operating point.

\section{ACKNOWLEDGMENT}

The authors acknowledge that this work has been supported by NSF grant CCF1302375.

\section{REFERENCES}

[1] T Austin. DIVA: a reliable substrate for deep submicron microarchitecture design. In Microarchitecture, 1999. MICRO-32. Proceedings. 32nd Annual International Symposium on, pages 196-207, 1999.

[2] R. Baumann. Soft errors in advanced computer systems. Design Test of Computers, IEEE, 2005.

[3] I. Bhati, et al. DRAM Refresh Mechanisms, Trade-offs, and Penalties. Computers, IEEE Transactions on, PP(99):1-1, 2015.
[4] C. Bienia, et al. The PARSEC Benchmark Suite: Characterization and Architectural Implications. In Proceedings of the 17th International Conference on Parallel Architectures and Compilation Techniques, PACT'08, pages 72-81, 2008.

[5] C. L. Chen and M. Y. Hsiao. Error-correcting Codes for Semiconductor Memory Applications: A State-of-the-art Review. IBM J. Res. Dev., 1984

[6] T. J. Dell. A white paper on the benefits of chipkill-correct ECC for PC server main memory.

[7] M. Hsiao. A Class of Optimal Minimum Odd-weight-column SEC-DED Codes. IBM Journal of Research and Development, 14(4):395-401, July 1970.

[8] G. Huang, et al. Power Delivery for 3D Chip Stacks: Physical Modeling and Design Implication. In Electrical Performance of Electronic Packaging, 2007 IEEE, pages 205-208, Octl 2007.

[9] W. Huang, et al. HotSpot: A compact thermal modeling methodology for early-stage VLSI design. TVLSI, 14(5):501-513, 2006

[10] R. Jayaseelan and T. Mitra. Temperature Aware Task Sequencing and Voltage Scaling. In Proceedings of the 2008 IEEE/ACM International Conference on Computer-Aided Design, ICCAD '08, pages $618-623,2008$.

[11] X. Jian, et al. Analyzing Reliability of Memory Sub-systems with Double-Chipkill Detect/Correct. In Dependable Computing (PRDC), 2013 IEEE 19th Pacific Rim International Symposium on, pages 88-97, Dec 2013.

[12] S. Li, et al. McPAT: an integrated power, area, and timing modeling framework for multicore and manycore architectures. In Microarchitecture, 2009. MICRO-42. 42nd Annual IEEE/ACM International Symposium on, pages 469-480. IEEE, 2009.

[13] J. Liu, et al. RAIDR: Retention-Aware Intelligent DRAM Refresh. SIGARCH Comput. Archit. News, 40(3):1-12, June 2012.

[14] G. H. Loh. 3D-Stacked Memory Architectures for Multi-core Processors. In Proceedings of the 35th Annual International Symposium on Computer Architecture, ISCA '08, pages 453-464, 2008.

[15] J. Meng, et al. Optimizing Energy Efficiency of 3-D Multicore Systems with Stacked DRAM Under Power and Thermal Constraints. In Proceedings of the 49th Annual Design Automation Conference, DAC'12, pages 648-655, 2012.

[16] P. Mercati, et al. Workload and User Experience-aware Dynamic Reliability Management in Multicore Processors. In Proceedings of the 50th Annual Design Automation Conference, DAC '13, pages $2: 1-2: 6,2013$

[17] J. Meza, et al. Revisiting Memory Errors in Large-Scale Production Data Centers: Analysis and Modeling of New Trends from the Field. In Dependable Systems and Networks (DSN), 2015 45th Annual IEEE/IFIP International Conference on, pages 415-426, June 2015.

[18] S. Mukherjee, et al. Detailed design and evaluation of redundant multi-threading alternatives. In Computer Architecture, 2002. Proceedings. 29th Annual International Symposium on, pages 99-110, 2002

[19] S. S. Mukherjee, et al. Cache Scrubbing in Microprocessors: Myth or Necessity? In Proceedings of the 10th IEEE Pacific Rim International Symposium on Dependable Computing (PRDC'04), PRDC '04, 2004 .

[20] J. S. Pak, et al. PDN Impedance Modeling and Analysis of 3D TSV IC by Using Proposed P/G TSV Array Model Based on Separated P/G TSV and Chip-PDN Models. TCPM, 1(2):208-219, Feb 2011.

[21] D. Roberts and P. Nair. Faultsim: A fast, configurable memory-resilience simulator. In The Memory Forum: In conjunction with ISCA, volume 41.

[22] K. Roy, et al. Leakage current mechanisms and leakage reduction techniques in deep-submicrometer CMOS circuits. Proceedings of the IEEE, 91(2):305-327, Feb 2003.

[23] B. Schroeder, et al. DRAM Errors in the Wild: A Large-scale Field Study. SIGMETRICS Perform. Eval. Rev., 2009.

[24] C. Serafy, et al. Unlocking the True Potential of 3-D CPUs With Microfluidic Cooling. TVLSI, PP(99):1-1, 2015.

[25] M. Shevgoor, et al. Quantifying the Relationship Between the Power Delivery Network and Architectural Policies in a 3D-stacked Memory Device. In Proceedings of the 46th Annual IEEE/ACM International Symposium on Microarchitecture, MICRO-46, pages 198-209, 2013.

[26] D. Skinner and W. Kramer. Understanding the causes of performance variability in HPC workloads. In Workload Characterization Symposium, 2005. Proceedings of the IEEE International, pages $137-149$, Oct 2005.

[27] T. Slegel, et al. IBM's S/390 G5 microprocessor design. Micro, IEEE, 19(2):12-23, Mar 1999 .

[28] V. Sridharan and D. Liberty. A Study of DRAM Failures in the Field. In Proceedings of the International Conference on High Performance Computing, Networking, Storage and Analysis, SC $12,2012$.

[29] R. Ubal, et al. Multi2Sim: a simulation framework for CPU-GPU computing. In Proceedings of the 21 st international conference on Parallel architectures and compilation techniques, pages 335-344. ACM, 2012.

[30] S. C. Woo, et al. The SPLASH-2 Programs: Characterization and Methodological Considerations. SIGARCH Comput. Archit. News, 23(2):24-36, May 1995. 